\title{
Research on Emergency Management of Pharmaceutical Care in Designated Hospital for Patients with COVID-19
}

\section{Xiu-lan Liu}

Tongji Hospital of Tongji Medical College of Huazhong University of Science and Technology Yi Liu

Tongji Hospital of Tongji Medical College of Huazhong University of Science and Technology Qiu Lin

Tongji Hospital of Tongji Medical College of Huazhong University of Science and Technology

\section{Luo Pan}

Tongji Hospital of Tongji Medical College of Huazhong University of Science and Technology Jin Jingjin

Tongji Hospital of Tongji Medical College of Huazhong University of Science and Technology

\section{Zheng Jianling}

Tongji Hospital of Tongji Medical College of Huazhong University of Science and Technology

\section{Dong Liu}

Tongji Hospital of Tongji Medical College of Huazhong University of Science and Technology

Juan Li (D947281063@qq.com )

Tongji Hospital of Tongji Medical College of Huazhong University of Science and Technology https://orcid.org/0000-0001-7070-1222

\section{Research article}

Keywords: COVID-19, Pharmacy administration, Pharmaceutical care, Clinical safety medication, Designated COVID-19 Hospital

Posted Date: April 5th, 2020

DOI: https://doi.org/10.21203/rs.3.rs-18380/v1

License: (c) (1) This work is licensed under a Creative Commons Attribution 4.0 International License.

Read Full License 


\section{Abstract}

Background: The novel coronavirus pneumonia (COVID-19) was entered into the critical period of epidemic prevention. Our hospital was designated as a hospital for severe pneumonia in Wuhan. Timely and effective pharmaceutical emergency support system is of great significance for the epidemic prevention and control of COVID-19.

Method:In order to ensure COVID-19 patients' medication needs and ensure the safety treatment, we focus on the key points and difficult problems in the practice of pharmaceutical management during the period of COVID-19, and then formulate appropriate pharmaceutical emergency support system combined with clinical practice.

Results:The pharmaceutical department quickly launched the emergency mechanism, formulated the key drug catalog for COVID-19, purchased some treatment drugs, reformed the emergency pharmacy process, established the donated drug management system, established the pharmacist consultation team, set up the "cloud pharmacy" for patients with chronic diseases other than COVID-19, and strengthened pharmacist protection management.

Conclusion:During the period of COVID-19, the pharmaceutical administration is a professional, comprehensive, complex and systematic emergency project to ensure the drug supply and safety administration.

\section{Background}

By the end of 2019, the novel coronavirus pneumonia [World Health Organization (WHO) recently named as Corona Virus Disease 2019 (COVID-19)] broke out in Wuhan and spread rapidly to the whole country[1]. On January 20, 2020, the National Health Commission issued No. 1 announcement, which included COVID-19 as an acute respiratory infectious disease into the class B infectious disease specified in the law of the people's Republic of China on the prevention and control of infectious diseases, and managed it as class A infectious disease. The COVID-19 epidemic situation was classified as "public health emergencies of international concern" in January 31st, the highest level in the WHO infectious disease emergency mechanism by $\mathrm{WHO}[2]$.

To guarantee the lives, health and safety of the people, Tongji Hospital affiliated to Huazhong University of Science and Technology (Tongji Hospital) reformed the medical area of 77,371 square meters in 15 days, and opened 2025 beds. 18 medical teams which formatted by respiratory and critical medicine department, ICU, cardiovascular medicine and other experts from Beijing, Changchun, Xi'an, Changsha and other places (Beijing Union Medical College Hospital, Peking University Third Hospital, Peking University People's Hospital, etc.) jointly carried out COVID-19 patients treatment work with Tongji Hospital. However, drugs demand for COVID-19 is uncertain, more than $85 \%$ of them are severe ill patients, involving almost all categories of drug management tasks, which is undoubtedly a huge challenge for hospital pharmacy work. Secondly, co-diagnosis and treatment, and most of the aid doctors 
are in critical care medicine, respiratory department, cardiovascular medicine and other specialties, pharmaceutical care need to be enhanced. The author summarizes the experience and lessons of Tongji Hospital for the treatment of COVID-19 patients, and proposes specific countermeasures, specifically for Pharmacy administration and pharmaceutical care so as to improve the ability of the hospital's pharmaceutical department to respond to large public health emergencies. The experience of pharmacy administration and pharmaceutical care of COVID-19 Designated Hospital is introduced as follows.

\section{Methods}

Based on regulations on emergency public health events, the methodology and general rules of WHO Guideline Development and the WHO Rapid Advice Guideline, pharmaceutical department quickly established $\square$ Emergency Management of Pharmaceutical Care for Patients with COVID-19凶. Emergency management theory was founded by Franz Edelman, the winner of the world's highest prize in management science. To analysis the key problem of management situation $₫$ we firstly applied the emergency management theory on pharmacy administration to solve human crisis, material crisis and responsibility crisis in Designated Hospital for Patients with COVID-19. In addition, The developed questionnaire was made accessible online on the drugs supply and selection of 18 medical teams.

\section{Results}

\section{Drug supply guarantee in COVID-19 designated hospital}

\subsection{Establish and improve the list of key therapeutic drugs for COVID-19}

Effective targeted treatment drugs have not yet confirmed for COVID-19. Based on the drugs included in Clinical Technical Guide issued by National and Provincial Health Commissions, the Ministry of Science and Technology, and relevant medical institutions for prevention, diagnosis and treatment, reference to the practice of designated hospital for severe ill patients[3], while the hospital was being renovated, determine the list of key therapeutic drug lists for COVID-19. Pharmacists conduct dynamic monitoring of key drug inventory and establish a shortage early warning and response mechanism, and timely report to the medical director of the pharmaceutical department and the drug procurement department to ensure the supply of key therapeutic drugs.

At the beginning of the epidemic condition, the Ministry of Pharmacy established the list of key therapeutic drugs including 180 kinds of medicines. According to the different basic diseases of the severe patients, drugs for the respiratory system, digestive system, cardiovascular system, blood and hematopoietic system, urinary and reproductive system, hormones and pediatrics were added. The list of related drugs has been expanded to 336. It contains 13 kinds of antiviral drugs such as Abidol, 
Lopinavir/ritonavir and Chloroquine; 29 kinds of antibacterial drugs such as third-generation cephalosporins, Moxifloxacin; 26 kinds of nutrition support drugs such as various types of fat milk; 25 kinds of proprietary Chinese medicines such as Lianhua Qingwen Capsule, Jinye Baidu Granule, Xuebijing Injection, Shengmai Injection, etc. In addition to a sufficient reserve of antiviral Chinese patent medicines, the supply of Chinese medicinal materials and frying-free granules is also guaranteed, which provides a guarantee for the development of syndrome differentiation and traditional Chinese medicine treatment on a one-on-one basis. Meanwhile, we will effectively protect the special needs of patients with cardiovascular disease, diabetes, children, and renal insufficiency including renal dialysis patients and maternal medication needs ( $T a b 1$ ), which provide better pharmaceutical cares to the patients.

\subsection{Establish emergency purchasing process}

In response to the sudden outbreak of COVID-19, the Pharmacy administrationand Pharmacotherapy Committee had urgently established a drug emergency procurement mechanism. For the Drug Products included in Centralized purchase catalogue of public hospitals in Huber Province, we will directly purchase it; for the drugs not included in the list, it will be purchased offline to protect the patient's treatment drugs in a timely manner. As of February 28, 2020, a total of 14 emergency procurement procedures have been initiated, including emergency purchases of a total of 26 medicines, such as Abidol, Lopinavir/ritonavir tablets, Ribavirin tablets, Ambroxol tablets, Zanamivir powder, Shengmai injection, Shenfu injection, Huoxiangzhengqi liquid and so on.

\subsection{Strengthen the Traditional Chinese Medicine production of self-prepared preparations}

Our hospital's preparation, Jinye Baidu Granule, is composed of honeysuckle, daqingye, dandelion and houttuynia cordata. It has antiviral, antipyretic, anti-inflammatory, antibacterial, endotoxin inhibition, and immunity enhancement functions. The effect for wind-heat lung heat in the treatment of fever, sore throat, cough, and expectoration is definite[4], and it proved to be effective in preventing and treating SARS virus infection during the SARS period. Based on the clinical practice, the COVID-19 specialist group of Wuhan, Hubei medical professional group and other experts concluded that the "Avidor + Jinye Baidu Granule" can be used as a recommended drug for the isolation period of patients. The Jinye Baidu Granule is included in the Hubei Provincial Health Committee's new control and storage medicine. At the beginning of the outbreak, the pharmacy department urgently arranged the preparation center to expand the production of Jinye Baidu Granule, and supply more antiviral Chinese medicine preparations into the marks. Subsequently, Guangdong, Hebei, Fujian and Anhui also listed it as a reserve drug for the prevention and control of the new crown epidemic condition. In addition, Yuxingcao nasal drops made by our hospital also have the effects of clearing away heat and detoxification and assisting in the treatment of virus infection, which are more suitable for cleaning the nasal cavity and ear cavity than disinfectants with strong stimulation. 
As of February 24, the preparation center of the Ministry of pharmacy has produced 99882 boxes of Jinye Baidu Granule, 38956 bottles of Yuxingcao nasal drops, 6280 bottles of $75 \%$ ethanol for disinfection, 5448 bottles of $3 \%$ hydrogen peroxide disinfectant, 23430 bottles of sterile purified water for auxiliary oxygen inhalation. The Preparation Center make-sure the continuous supply of common drug and effective disinfectants for COVID-19.

\subsection{Transform pharmacy process from PIVAS to emergency pharmacy for COVID-19}

The ward environmental cleanness in designated COVID-19 hospital cannot meet infusion configuration. Secondly, most of the patients in our hospital are severe patients, and the nursing staffs have heavy work[5]. In addition, the nursing staffs are limited in action for wearing protective clothing, which is difficult to meet the requirements of accurate infusion configuration. For this reason, the Pharmacy Intravenous Admixture Service (PIVAS) centralized configuration mode must be adopted[6].

The pharmaceutical department quickly investigated and integrated the pharmacy of the original inpatient department and PIVAS into the emergency pharmacy, and formulated the PIVAS node control process of "first review and fifth check", covering all links of the order review, allocation, check, labeling, warehousing, mixed allocation, finished product review, sorting and packaging, and infusion distribution of intravenous infusion, strictly checking at all levels to ensure the quality of each bag of finished infusion. Focus on the audit of medical orders, based on the support of the big data information system of Meikang system, realize the pre intervention of medical orders. At the beginning of the outbreak, the treatment is in the exploratory stage, so the over adaptation and overdose of drugs are not the targets of software interception, only record and give suggestions, but focuses on the serious drug interaction and the incompatibility of infusion, so as to better guarantee the drug safety of patients.

In addition, for non-venous drugs, such as oral administration, atomization inhalation, external use, etc., pack and distribute in the unit of the ward; for patients who are cured and discharged, discharge medication is carried out from the inpatient order, and discharge medication is no longer issued separately to avoid possible cross infection, as shown in Figure 1.

\subsection{Transformation of drug distribution process to avoid latrogenic infection}

In order to avoid iatrogenic infection and improve efficiency, we transformed drug distribution process to paperless prescriptions in fever clinics, as follows, the system automatically generated electronic prescriptions and imported CA electronic signature of doctors, after pharmacists received and checked it, the system automatically imported CA electronic signature of pharmacists. Subsequently, we applied paperless prescriptions to management of special drugs in inpatient pharmacy. Drug prescriptions such as narcotic drugs, class I psychotropic drugs and drugs for termination of pregnancy are paperless 
prescribed, and the system automatically introduces electronic signatures of doctors and pharmacists to generate special prescriptions for future reference.

\subsection{Adjust the standby drugs in the ward and ensure drugs timely for the severe patients}

Our hospital is a designated hospital for severe patients. More than $85 \%$ of the patients are critically ill. The type and quantity of standby drugs in the ward is very important to ensure the timeliness of treatment. Collect the demand of "standby drugs" from clinical departments in the ward, and formulate the catalog and base number of standby drugs in COVID-19 ward. It includes first-aid drugs such as antishock vasoactive drugs, muscle relaxants and sedatives, and non-first-aid drugs, as shown in Table 2 . For ICU departments, the number of standby drugs should be increased to 200 to ensure the urgently needed drugs of severe patients. In addition, according to the management system of standby drugs in the ward of our hospital, a special person shall be appointed to the ward every month to check the drug management. For special drugs such as narcotic drugs, a "registration book for use of narcotic drugs in the ward" shall be established and the use records shall be improved.

\subsection{Improve the management process of clinical trial medication and guarantee the demand of experimental medication}

In accordance with the relevant requirements of Measures for the administration of drug registration, the code for the quality management of drug clinical trials and the measures for the supervision and administration of drug research 9 (Trial) issued by the State Food and drug administration, the standardized management of clinical trials in our hospital is standardized. There's no special drug for COVID-19. For this reason, our hospital has opened a green channel for the COVID-19 clinical trial. The ethics committee has accelerated the approval process. Strict management mode is adopted for the trial drug. The purchase and account management are carried out in accordance with the normal flow management process in the hospital. The pharmaceutical department is responsible for the quality control management of drugs.

\subsection{Set up "cloud pharmacy" to meet the drug demand of non COVID-19 chronic disease patients}

During COVID-19, $90 \%$ of the medical institutions were all ordered to be admitted as the designated hospitals, and there is no guarantee for medication for patients with chronic diseases. In February 14, 2020 , in order to facilitate the public's orderly diagnosis and treatment, convenient drug use and avoid cross infection[7], our hospital established "cloud clinic" to provide online video inquiry, set up cloud pharmacy. Pharmacists audited "cloud prescription" after deployment check, drug distribution one-stop care function. As of March 13, 14900 online video consultations had been conducted, and 11642 prescriptions had been prepared for non COVID-19 patients, covering people with hypertension, diabetes, cerebrovascular diseases, etc. 


\section{Pharmaceutical care in designated hospital for COVID-19}

\subsection{Formulation of COVID-19 treatment agents and solvents}

The treatment of COVID-19 is often combined with multiple drugs, even with some clinical data and evidence lacking evidence-based medicine, therefore, drug interactions and incompatibility should be fully emphasized. Some medical staff may be not familiar with the compatibility of drugs for COVID-19 and common solvent. The pharmacy department quickly formulated the COVID-19 treatment related drug interaction search table, which has covered 76 kinds of intravenous infusion drugs, "intravenous drug for COVID-19 and common solvent compatibility table, which has 13 kinds of common treatment drugs. The front-line medical staff can quickly consult and use to ensure clinical safety medication.

\subsection{Participate in clinical consultation and improve pharmaceutical care}

Our hospital is a designated hospital for severe ill patients. Most of them are complicated with chronic basic diseases such as diabetes, cardiocerebrovascular disease, respiratory disease, etc. In order to better ensure the clinical safety of medication, the pharmaceutical department appoints specialized clinical pharmacists to participate in the consultation of difficult cases in the ward and the daily case discussion in the whole hospital, and analyzes the death cases 2-3 times a week. For special populations[8], such as those with liver and kidney dysfunction, pregnant women, children, etc., pharmacists are more cautious when recommending medications, fully fellow the concept of evidence-based pharmacy, and choose the most appropriate medicine. As of February 28, 2019, we have participated in 20 multidisciplinary consultations, provided drug treatment recommendations, realized drug reformation, and reduced the risk of drug interactions and adverse reactions.

\subsection{Pay attention to the use of key drugs and monitor adverse drug reactions}

There's no effective treatment drug for COVID-19, Drugs recommended by COVID-19 treatment plan (Trial Seventh Edition), such as Chloroquine and Abdor are mostly beyond the prescribed medication. The pharmacy department has issued guidelines for rational use of drugs for the treatment of COVID-19, such as Lopinavir / litonavir, Ribavirin, Chloroquine, Abidol, Tosubzumab and so on. Special personnel shall be arranged to monitor the adverse drug reactions such as Chloroquine, Lopinavir / ritonavir, Abidol, etc. In addition, the pharmaceutical team focuses on the comprehensive pharmaceutical care of severe patients, and increases efforts to carry out adverse drug reaction monitoring, especially for the new treatment drugs, the old drugs with new indications, the combination of Chinese and Western drugs and other 
situations[9]. As of February 28, 2019, a total of 39 adverse drug reactions have been reported, and clinical medications have been followed in real time to ensure the safety of patients' medications.

\subsection{Write Popular science knowledge about COVID-19 and improve public awareness}

Drug publicity and education under the mode of informational pharmaceutical care has always been a pharmaceutical care that pharmacists need to do well for a long time. In order to reduce the flow of people and improve the efficiency of medication education, pharmacists will play the common medication precautions and education on drug use after discharge in the form of video.

In order to eliminate the panic among the people as soon as possible and popularize the knowledge of COVID-19 treatment drugs, the Ministry of Pharmacy organized pharmacists to write popular sciencerelated medicines and publish them on the WeChat public account of the Ministry of Pharmacy, the WeChat public account of the hospital and the official website to reduce blindly purchase of drugs and misuse of drugs, promote patients' understanding of the diagnosis and treatment plan, and cooperate with medical staff to actively treat or self-treat. The articles were reproduced by the public.

\section{Construction of donated drug management system}

During the epidemic period, the number of donated drugs and disinfectants received by Tongji Hospital increased rapidly. With reference to the management experience of donated drugs in the period of SARS and Wenchuan earthquake and combined with the actual situation, the emergency management plan for donated drugs and disinfectants in Tongji Hospital was established to improve the management process of donated drugs and disinfectants. So as to ensure that the source of drugs is legal, the quality is reliable, the destination is traceable and transparent.

When purchasing or receiving the donated disinfectant, the donor is required to provide the relevant qualification of the production enterprise, the Certificate of the donation batch and other documents, and receive the donated materials that meets the requirements of inactivating the new coronavirus according to the regulations of the hospital infection management department, so as to meet the needs of medical disinfection. The pharmaceutical department, the infection management department and the nursing department of the hospital jointly establish the standard operating procedures for the use of hospital disinfectants, establish standardized method of preparations, and ensure that the effective ingredients and contents of disinfectants meet the needs in the epidemic period.

For donated drugs, it should be warehoused with zero price, to ensure that donated drugs benefit patients and front-line medical staff. At the same time, in order to ensure the transparency of the donation materials, patients need to issue medical orders for use, and medical staff use the anti-epidemic as the unit, and get the money through the temporary fund card, as shown in Table 3. 


\section{Establishment of pharmacist protection and monitoring system}

The Ministry of Pharmacy has established a personnel protection training mechanism that monitors the health of personnel daily, including body temperature monitoring, cough, diarrhea, hand hygiene, oral cavity, ear canal and auricle, and nasal cavity hygiene[10]. For high-risk positions such as hot clinics and pharmacies, fellow two-level protection standards, standardize the procedures on putting on and putting of the protective clothing, goggles, face shields and other protective equipment, and use a video surveillance system to dynamically monitor; For low-risk positions, such as intravenous infusion configuration centers, on the basis of primary protection standards, wear isolation clothing; for drug stores, offices, etc., fellow the primary protection standard.

\section{Discussion}

Pharmacy administration should combine the characteristics of the pharmacy profession to establish a pharmacy emergency guarantee mechanism that meets the actual needs of medical treatment[11]. COVID-19 and practices of pharmacy administration common to emergencies such as earthquakes and floods have in common the need to store a large number of disinfection drugs such as chlorinecontaining disinfectants, $75 \%$ alcohol, peracetic acid and hydrogen peroxide solutions. The differences are as follows: First, the list of key therapeutic drugs is different. In this case, the focus is on COVID-19 drugs and drugs for severe ill patients such as diabetes, cardio-cerebral vascular disease, kidney disease and respiratory diseases[12]. The earthquake focused on drugs for trauma such as fractures, craniocerebral injury and skin diseases, while floods focus on drugs for digestive system and skin diseases.

Secondly, the knowledges about COVID-19 is in the investigative phase. The damage of the virus to the organs of the body is gradually recognized. The therapeutic drugs need to be updated in time with the relevant diagnosis and treatment guidelines[13], and the earthquake disaster, flood and other disasters have sufficient knowledge and relevant experience to learn.

This paper mainly discusses fully guarantee of drugs supply during the period of COVID-19 in Pharmaceutical Department of our hospital, formulates the catalogue of key therapeutic drugs of COVID19 , urgently purchases some therapeutic drugs, transforms the process of intravenous infusion distribution center, builds the management system of donated drugs, sets up the consultation team of pharmacists, provides pharmaceutical care, fills in the short board of specialized drug knowledge of rescue medical team and improve the quality of pharmaceutical care. In addition, the establishment of "cloud pharmacy" to meet the needs of non COVID-19 patients with chronic diseases, arrange the patients overall, strengthen the connection, so that patients' drug treatment and drug safety are guaranteed, and the treatment work is carried out orderly. 


\section{Conclusions}

Finally, according to the characteristics of pharmacy department, establishing a standardized, hierarchical protection mechanism for pharmacists, and strengthening training and monitoring are also the focus during the period of COVID-19.

Due to the lack of relevant protective conditions, the blood drug concentration monitoring project has not been launched. It is recommended that the construction of a blood drug concentration monitoring laboratory should consider the detection of blood samples from infectious diseases. At present, our hospital is still treating patients with COVID-19, so there is no presentation of drug reprocessing after the epidemic conditions. The pharmaceutical management practice in designed hospital for severe ill patients with COVID-19 will provide reference for future large-scale public health events.

\section{Abbreviations}

COVID-19: Corona Virus Disease 2019; SARS: Severe Acute Respiratory Syndrome; WHO: World Health Organization; PIVAS: Pharmacy Intravenous Admixture Service.

\section{Declarations}

The authors declare that they have no competing interests.

\section{Acknowledgements}

We appreciate the healthcare workers fight in the front line of the outbreak of COVID-19.

\section{Funding}

Not applicable.

\section{Ethics approval and consent to participate}

The ethics committee of the University of Science and Technology (HUST) ruled that no formal ethics approval was required in this case, as no human subjects or other animals were involved in the study.

\section{Availability of data and materials}


All data generated or analysed during this study are included in this published article.

\section{Authors' contributions}

LJ, DL and XLL performed the study design and revised the manuscript. YL, LQ, JJJ, JLZ, PLmade substantial contributions to the collection of data. All authors read and

approved the final manuscript.

\section{Consent for publication}

Not applicable.

\section{References}

1. Organization WH. Clinical management of severe acute respiratory infection when novel coronavirus ( 2019-nCoV) infection is suspected: interim guidance, 28 January 2020. World Health Organization; 2020.

2. Lai C-C, Shih T-P, Ko W-C, Tang H-J, Hsueh P-R. Severe acute respiratory syndrome coronavirus 2 (SARS-CoV-2) and corona virus disease-2019 (COVID-19): the epidemic and the challenges. Int $J$ Antimicrob Agents. 2020;:105924.

3. Liang P-H. Characterization and inhibition of SARS-coronavirus main protease. Curr Top Med Chem. 2006;6:361-376.

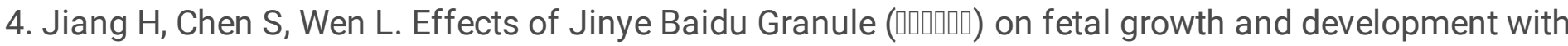
maternal active human cytomegalovirus infection. Chin J Integr Med. 2006;12:250-254.

5. QIU F, LIN C. Control Measures and Experience of Air Purification Management in PIVAS of Our Hospital [J]. China Pharm. 2012;5.

6. GU Y, ZHU X, DONG M. Analysis of the Rationality of Intravenous Medication in PIVAS of Cancer Hospital. China Pharm. 2013;2013:4.

7. Narayanan HAJ, Güneş MH. Ensuring access control in cloud provisioned healthcare systems. In: 2011 IEEE Consumer Communications and Networking Conference (CCNC). IEEE; 2011. p. 247-251.

8. ZHANG Z, CAO W, JIANG H. Exploration of "Specialization and Consultation" Pattern for Clinical Pharmacists in Our Hospital [J]. China Pharm. 2012;42. 
9. Sangasapasviliya A, Prakongwong T, Ayuthaya PK. Drug Hypersensitivity in Phramongkutklao Hospital. J Med Assoc Thai. 2010;93 Suppl 6:S106-11.

10. Zhang X, Zheng Q, Lv Y, An M, Zhang Y, Wei Y, et al. Evaluation of adverse health risks associated with antineoplastic drug exposure in nurses at two Chinese hospitals: the effects of implementing a pharmacy intravenous admixture service. Am J Ind Med. 2016;59:264-273.

11. Long SS, Pickering LK, Prober CG. Principles and practice of pediatric infectious disease. Elsevier Health Sciences; 2012.

12. Zhou F, Yu T, Du R, Fan G, Liu Y, Liu Z, et al. Clinical course and risk factors for mortality of adult inpatients with COVID-19 in Wuhan, China: a retrospective cohort study. 2020.

13. Li G, De Clercq E. Therapeutic options for the 2019 novel coronavirus (2019-nCoV). Nature Publishing Group; 2020.

\section{Tables}

Due to technical limitations, tables are only available as a download in the supplemental files section.

\section{Figures}




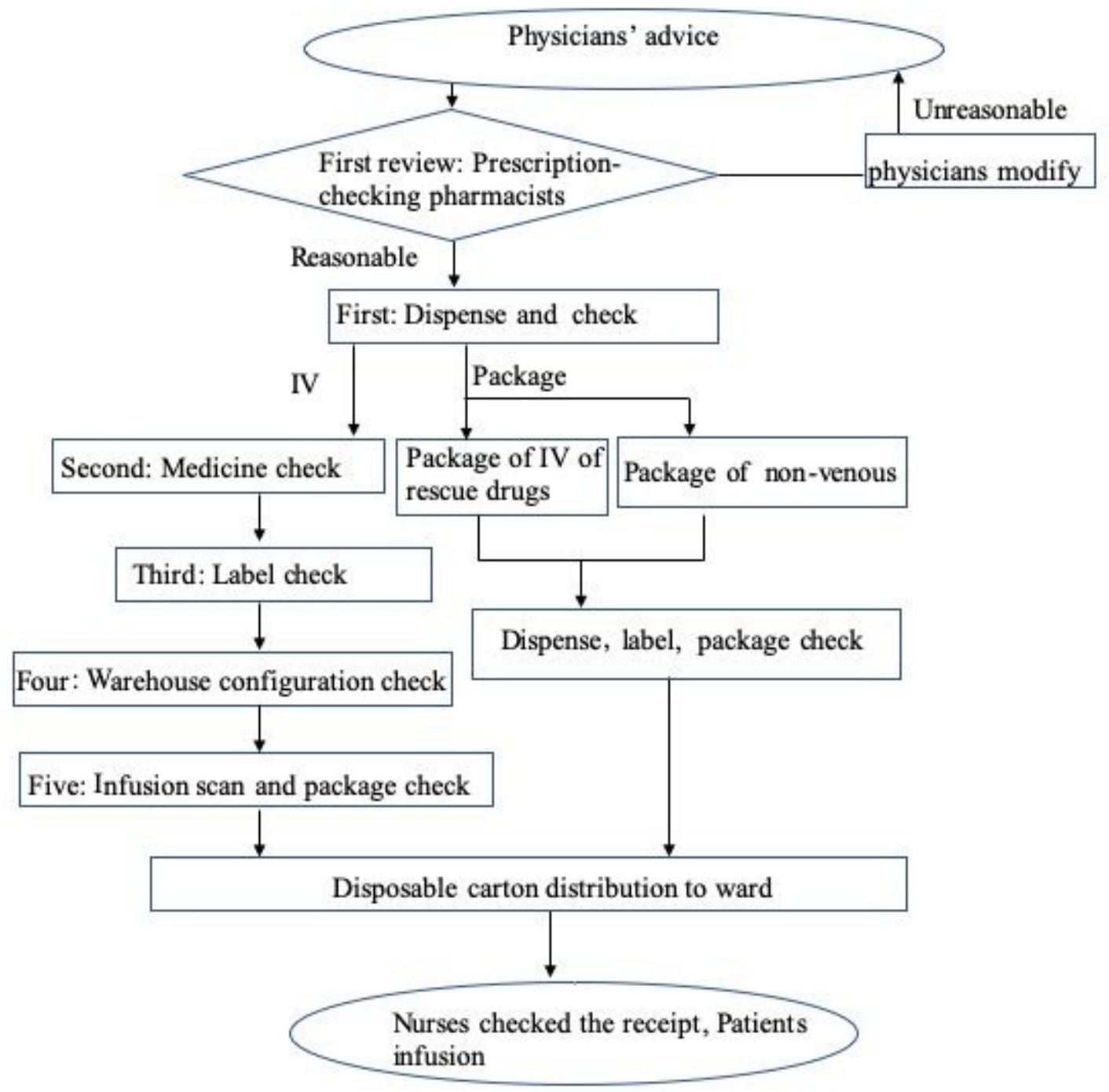

Figure 1

Process improvement of emergency pharmacy for COVID-19 The pharmaceutical department transformed pharmacy process from PIVAS to emergency pharmacy for COVID-19, and then, formulated the PIVAS node control process of "first review and fifth check". For non-venous drugs, the drugs would be packed and distributed in the unit of the ward.

\section{Supplementary Files}

This is a list of supplementary files associated with this preprint. Click to download.

- Table2.docx

- Table3.docx

- Table1.docx 